\title{
Variability in the therapeutic management of advanced ovarian cancer patients: a five-country survey of oncologists
}

This article was published in the following Dove Press journal:

Drug Design, Development and Therapy

\section{Céline Audibert ${ }^{\prime}$ \\ Anna Perlaky² \\ Mark Stuntz ${ }^{2}$ \\ Daniel Glass ${ }^{2}$}

'Deerfield Institute, Epalinges, Switzerland; ' ${ }^{2}$ Deerfield Institute, New York, NY, USA
Correspondence: Céline Audibert Deerfield Institute, Route de la Corniche 3a, 1066 Epalinges, Switzerland

$\mathrm{Tel}+4|2165| 9084$

Email caudibert@deerfield.com
Background: Advanced ovarian cancer patients have a poor prognosis, mainly because the disease is diagnosed at a late stage. A number of therapeutic approaches, such as neoadjuvant and maintenance therapies, have been developed to try to improve treatment outcome. In parallel, the targeted therapies bevacizumab and olaparib have recently been approved for ovarian cancer treatment. The goal of our survey was to provide a comprehensive, global depiction of advanced ovarian cancer treatments across different regions.

Patients and methods: Oncologists from France, Italy, Germany, the UK, and the USA were invited to participate in an online survey. Participants were eligible if they personally managed at least 15 ovarian cancer patients. Quantitative questions addressed the proportion of patients in neoadjuvant, treatment, and maintenance settings; proportion of BRCA-positive patients; and the type of treatment prescribed per setting and per line of therapy, depending on the patient's $B R C A$ status.

Results: A total of 138 respondents completed our survey in Europe and 132 in the USA. The proportions of patients in treatment, maintenance, and remission were identical across each country and line of treatment at $60 \%, 20 \%$, and $20 \%$, respectively. The proportion of $B R C A$ tested patients ranged from $45 \%$ in Italy to $73 \%$ in the USA, with $10 \%$ (UK)- $21 \%$ (Italy) of tested patients having a positive status. Levels of bevacizumab and olaparib prescriptions differed based on the country, line of treatment, and setting, with a significant share of patients receiving both drugs outside of their approved indications for ovarian cancer treatment.

Conclusion: This survey provides real-world data on how advanced ovarian cancer patients are currently treated: 1) $B R C A$ testing was not performed systematically, which raises concerns regarding access to treatment and 2) absence of consensus regarding which chemotherapeutic regimens or targeted therapy to use in different stages of the disease.

Keywords: advanced ovarian cancer, $B R C A$ mutation, maintenance, treatment, targeted therapies

\section{Introduction}

Ovarian cancer incidence is estimated at 238,000 patients worldwide per year, making it the seventh most common type of cancer among women. ${ }^{1}$ In the USA, epithelial ovarian cancer is the fifth most common cause of cancer mortality among women, and in 2017 , it is estimated that 22,440 new diagnoses and 14,080 deaths from this neoplasm will occur. $^{2}$ A number of characteristics, such as family history of ovarian cancer, reproductive history, and $B R C A$ mutation profile, have been shown to play a role in a woman's risk of developing ovarian cancer. ${ }^{3-5}$ Thirty-nine percent of women with a BRCA1 mutation and $11 \%-17 \%$ of women with a BRCA2 mutation will develop ovarian cancer by 
70 years of age. ${ }^{3,4}$ Prognosis for ovarian cancer patients is poor, predominantly because it is diagnosed at a late stage when the disease is already widely metastatic, with $75 \%$ of women with ovarian cancer being diagnosed at Stage IIIC or IV. ${ }^{6,7}$

The standard of care consists of cytoreductive surgery and postoperative or adjuvant chemotherapy. ${ }^{8}$ Platinum-based chemotherapy has been the backbone of ovarian cancer treatment and is used until the tumor becomes resistant. ${ }^{8}$ Advanced ovarian cancer patients receive multiple lines of therapy, with ever-decreasing period of treatment-free times. ${ }^{8}$ Attempts to improve patients' survival include the use of neoadjuvant chemotherapy when complete debulking is not feasible, ${ }^{9-11}$ intraperitoneal treatment, ${ }^{12,13}$ and use of maintenance therapy. ${ }^{14}$

The most recent changes in ovarian cancer treatment are the approval of targeted therapies such as bevacizumab and olaparib. ${ }^{15,16}$ Bevacizumab, first approved by the European Medicines Agency in 2011, is a monoclonal antibody that binds to all isoforms of the vascular endothelial growth factor receptor ligand. ${ }^{17}$ A number of studies showed that use of bevacizumab, either alone or in combination with chemotherapy, could be beneficial in various treatment settings such as front line, recurrent patients, platinum-sensitive, and platinumresistant patients. ${ }^{18-22}$ The US Food and Drug Administration (FDA) initially approved bevacizumab in combination with chemotherapy for the treatment of platinum-resistant ovarian cancer patients in November 2014. ${ }^{15}$

Olaparib is a poly (ADP-ribose) polymerase inhibitor that induces synthetic lethality in BRCA1/2-deficient tumor cells. ${ }^{23}$ In December 2014, the FDA approved olaparib for patients with advanced ovarian cancer who received treatment with three or more lines of chemotherapy and who have a germline $B R C A$ mutation, while the European Medicines Agency approved it as monotherapy for the maintenance of platinum-sensitive relapsed $B R C A$-mutated patients whose previous treatment with platinum-based chemotherapy led to a sustained response. ${ }^{16,24}$

In this context, a multi-country survey was designed to investigate current treatment of advanced ovarian cancer patients. More specifically, this survey investigated the proportion of patients in treatment versus those in maintenance, and the type of treatment received depending on their $B R C A$ mutation status. To our knowledge, this was the first research investigating real-world pharmacologic therapy prescribed to ovarian cancer patients in multiple countries.

\section{Patients and methods}

\section{Study sample design}

Medical and gynecologic oncologists from France, Germany, Italy, the UK, and the USA were invited to participate in an online survey aimed at better understanding the medical management and treatment regimens of advanced stage epithelial ovarian cancer, in both $B R C A$-positive and -negative patients.

In the USA, a universal sample frame of ovarian cancertreating oncologists was created by sourcing Symphony Health Analytics' 2016 insurance claims activity for medical and gynecologic oncologists in the USA for the C56 series of International Classification of Diseases-10 codes used in diagnosing and treating ovarian cancer. Overall, 4,219 oncologists with activity related to the care of ovarian cancer patients were identified. This list of oncologists served as the sample frame for this survey and they were invited to the survey. An external provider, M3 Global Research, was used in Europe to contact medical and gynecologic oncologists. M3 Global Research is a panel provider which has access to a broad range of physicians, including those who specialize in gynecologic cancer treatment. A total of 369 medical and gynecologic oncologists were contacted in France, 551 in Germany, 340 in Italy, and 669 in the UK. Oncologists were eligible to participate if they personally managed at least 15 ovarian cancer patients at the time of survey participation. Altogether, 361 European oncologists responded to the survey and 138 met the eligibility criteria and completed the survey. In the USA, a total of 188 oncologists responded to the survey, with 132 meeting the eligibility criteria. Response rate could not be calculated since the proportion of emailed physicians who fit in the inclusion criteria or who have an active email address is unknown.

Participants were offered an industry-standard honorarium as compensation for their time in completing the survey. The survey was administered online and was fielded from December 16, 2016 to January 23, 2017.

\section{Survey design}

A questionnaire was developed to collect anonymized information on patients with advanced ovarian cancer. We developed and pretested this instrument through interviews and consultations with three ovarian cancer-treating oncologists before launching the survey online. The wording of the questions and their order were carefully decided to minimize any influence that one question may have on subsequent questions. The online questionnaire consisted largely of quantitative questions, and covered the following topics: patients' disease stages; proportion of $B R C A$-tested patients; outcome of $B R C A$ tests and reasons for not testing; proportion of patients per line of therapy; management of advanced ovarian cancer patients per line of therapy depending on their $B R C A$ status; and whether they were on 
chemotherapy or maintenance therapy (questionnaire available upon request).

\section{Data analysis}

All survey data were analyzed in aggregate, and the study authors were blinded to the individual identities of the survey respondents. The planned analyses for quantitative data were descriptive and included means and percentages. Data from each respondent were weighted by the total number of advanced ovarian cancer patients they see to account for the differences between large and small practices. No formal statistical tests were performed. Qualitative data were analyzed thematically and coded according to the main themes of the survey questions. Any response that addressed multiple themes was counted as multiple comments.

\section{Ethics}

By electing to complete the survey, respondents provided consent to use their anonymous responses to the survey questions. The study did not involve patients, and data on patient characteristics were provided only in aggregate. As such, there was no institutional review board and/or licensing committee involved in approving the research and no need for informed consent from the participants as per the US regulations edited by the US Department of Health and Human Services, Protection of Human Subjects 45 CFR $\$ 46.101$, paragraph (b) (4)..$^{25}$

\section{Results}

A total of 549 physicians responded to the survey invitation and 270 met the eligibility criteria and completed the survey ( $49 \%$ completion rate). The number of respondents by country who completed the survey included 138 oncologists in Europe ( 37 in France, 37 in Germany, 31 in Italy, and 33 in the UK), and 132 in the USA.

\section{Characteristics of treated ovarian cancer patient population}

The mean number of treated ovarian cancer patients per respondent was 36.7 in Italy, 46 in Germany, 51.2 in the USA, 64 in France, and 86.8 in the UK. Stages III and IV represented the vast majority of patients, ranging from $65 \%$ in Italy to $81 \%$ in the USA. Germany, Italy, and the USA had the lowest proportion of ovarian cancer patients in neoadjuvant treatment $(16 \%, 15 \%$, and $16 \%$, respectively) versus $22 \%$ in the UK and $26 \%$ in France. Germany had the highest proportion of patients in first-line treatment at $44 \%$, compared with $30 \%-36 \%$ in all other countries (Table 1). In terms of the type of treatment, $60 \%$ of advanced ovarian
Table I Proportion of patients per line of treatment per country

\begin{tabular}{llllll}
\hline Country & $\begin{array}{l}\text { Number of } \\
\text { ovarian cancer } \\
\text { patients } \\
\text { (mean) }\end{array}$ & $\begin{array}{l}\text { Stage I, Stage II, Stage III, Stage IV, } \\
\%\end{array}$ & $\begin{array}{l}\% \\
\%\end{array}$ \\
\hline France & 64 & 10 & 14 & 32 & 45 \\
Germany & 46 & 9 & 14 & 40 & 37 \\
Italy & 37 & 16 & 19 & 33 & 32 \\
UK & 878 & 10 & 14 & 36 & 40 \\
USA & 51 & 9 & 9 & 52 & 29 \\
\hline
\end{tabular}

Notes: Based on 37 oncologists in France, 37 in Germany, 3 I in Italy, 33 in the UK, and $I 32$ in the USA.

cancer patients were on active treatment chemotherapy, 20\% were on maintenance therapy, and $20 \%$ were not currently treated. This split was observed for all lines of treatment, and there were no meaningful country-level differences (Figure S1). Resistance or absence of response to platinumbased therapy increased with the line of treatment, ranging from $19 \%$ in Germany and the USA to $33 \%$ in Italy in first line. In the fourth line or above, resistance or absence of response to platinum-based chemotherapy ranged from $49 \%$ to $65 \%$ (Figure $\mathrm{S} 2$ ).

\section{$B R C A$ mutation status}

The proportion of patients tested for $B R C A$ mutations was $45 \%$ in Italy, $50 \%$ in the UK, $61 \%$ in France, $65 \%$ in Germany, and $73 \%$ in the USA. The main reasons for not testing patients for $B R C A$ mutations were country specific: absence of family history or risk factor was the main reason in Italy $(28 \%)$, Germany (34\%), and the UK (43\%). In Italy, both the absence of family history or risk factor and the patient's condition preventing a biopsy were mentioned (28\% each). In France, the patient's condition preventing a biopsy was the main barrier (35\%), while in the USA, it was mainly the patient's choice not to be tested (49\%).

Of the patients who were tested for BRCA mutations, $10 \%$ had a positive test in the UK, $13 \%$ in France and Germany, $16 \%$ in the USA, and $21 \%$ in Italy.

\section{Pharmaceutical management of $B R C A$-negative patients}

Figure 1 shows how $B R C A$-negative patients were treated in each country. While platinum-based chemotherapy was prescribed the most in first line in the USA, UK, Italy, and France (ranging from 55\% to $93 \%$ of patients), German doctors preferred a combination of bevacizumab and chemotherapy ( $53 \%$ of patients). Shares of platinum-based chemotherapy decreased as patients progressed through treatment line, to the benefit of non-platinum-based chemotherapies. There was 
A

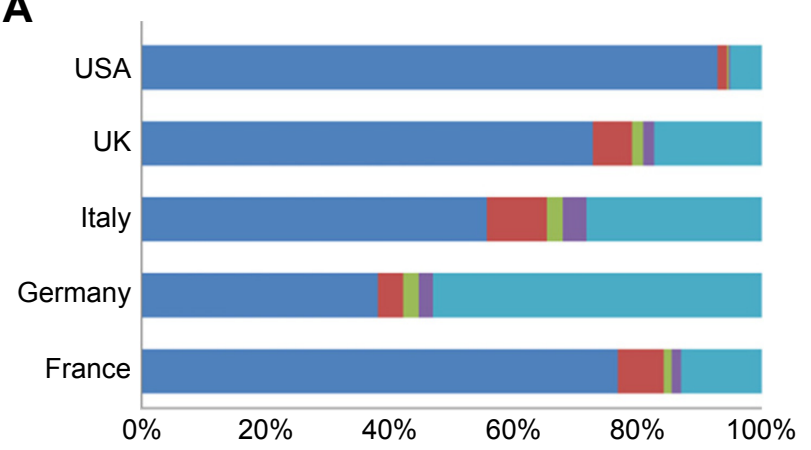

C

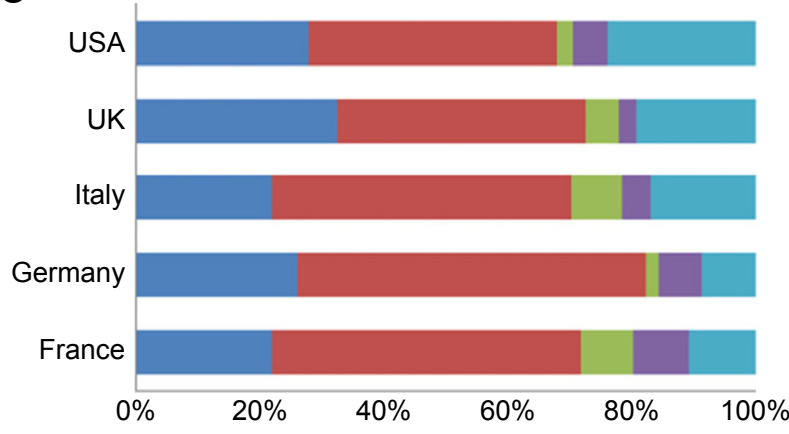

B

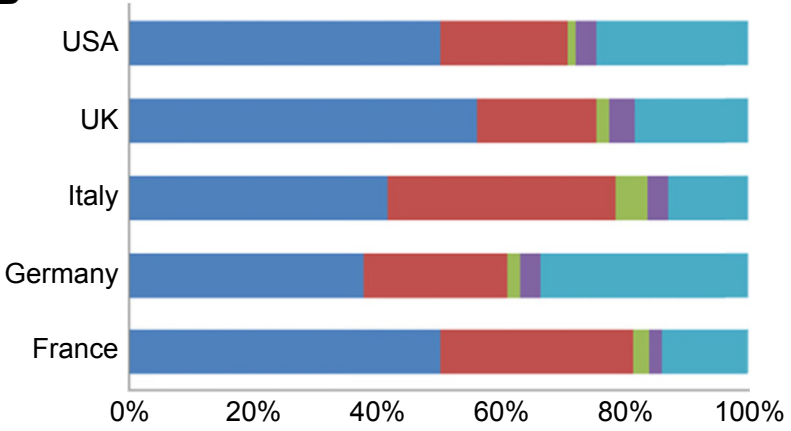

D

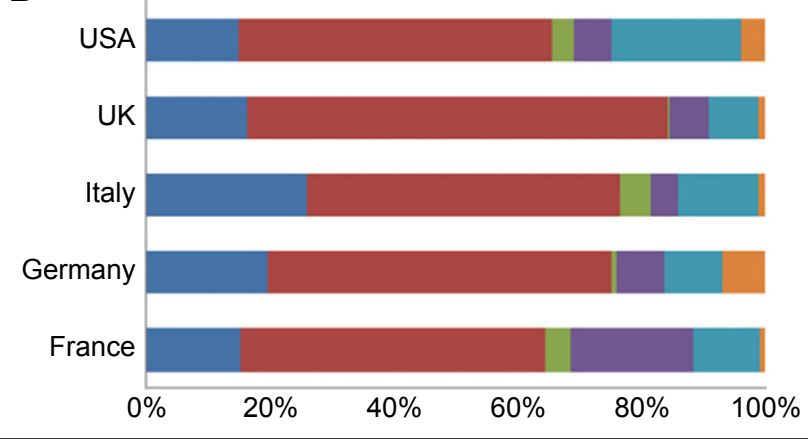

Platinum-based chemo $\square$ Non-platinum based chemo $\square$ Olaparib $\square$ Bevacizumab mono $\square$ Bevacizumab + chemo $\square$ Other

Figure I Treatment of BRCA-negative ovarian cancer patients.

Notes: Proportion of patients split by country and type of therapy: (A) first-line treatment, (B) second-line treatment, (C) third-line treatment, (D) fourth-line treatment or more.

no consensus regarding the use of bevacizumab; its shares varied per country and per line of treatment.

For maintenance of $B R C A$-negative patients, prescriptions were largely dominated by bevacizumab in monotherapy in first and second line. The proportion of patients receiving non-platinum-based chemotherapy increased in the third and fourth line (Figure 2).

Of note, some use of olaparib was observed in both the treatment and maintenance setting of $B R C A$-negative patients, when olaparib was indicated in $B R C A$-positive patients only at the time of the survey (Figures 1 and 2).

\section{Pharmaceutical management of $B R C A$-positive patients}

Similar to $B R C A$-negative patients, $B R C A$-positive patients were largely treated with platinum-based chemotherapy in first line in the USA, UK, Italy, and France, while German doctors predominantly prescribed bevacizumab in combination with chemotherapy (Figure 3). Use of platinum-based chemotherapy decreased as patients recurred, with a switch toward non-platinum-based chemotherapy. Prescription of olaparib varied per country and per line of treatment: $3 \%$ of patients received it in first line in the USA compared with
$22 \%$ in France. Although olaparib was only approved in the maintenance setting in Europe, all four European countries included in our survey reported using olaparib in the treatment setting.

Regarding maintenance of $B R C A$-positive patients, prescriptions of olaparib and bevacizumab represented a large proportion of patients in France, Germany, and Italy for all treatment lines (Figure 4). In the UK, chemotherapy (either platinum based or non-platinum based) represented the majority of treatments in first and second line, with a switch to olaparib and bevacizumab in third and fourth line. In the USA, olaparib was used for the maintenance of $B R C A$ positive patients in all lines of treatment, despite its approval in the treatment setting only.

\section{Discussion}

The management of advanced ovarian cancer has rapidly changed in the past 2 years with the approval of targeted therapies such as bevacizumab and olaparib. ${ }^{15,16} \mathrm{We}$ designed a survey to investigate the real-world current treatment practices in the therapy and the maintenance setting, and depending on patients' $B R C A$ status, in the USA and four European countries. 
A

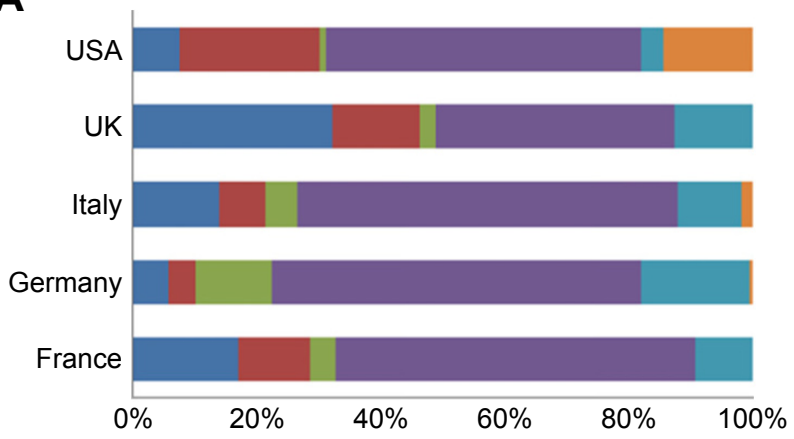

C

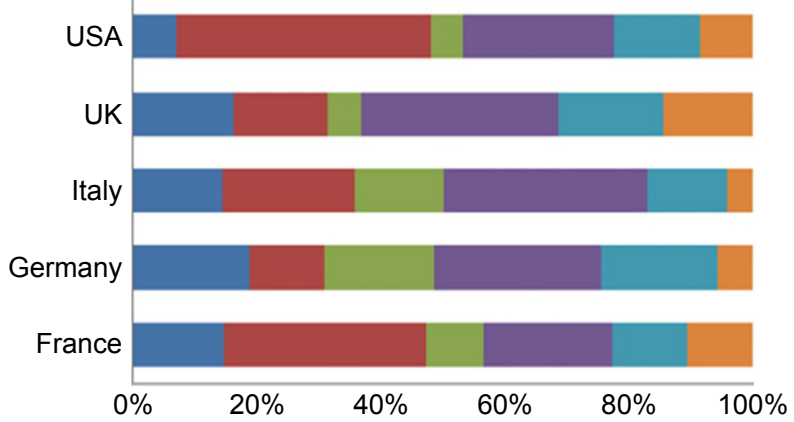

B

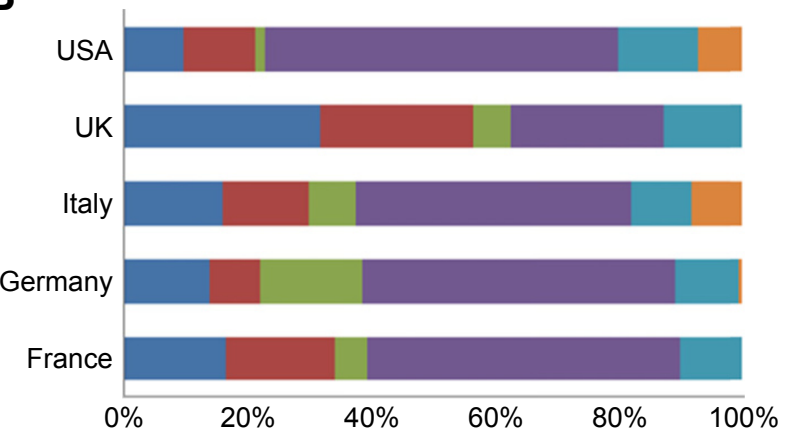

D

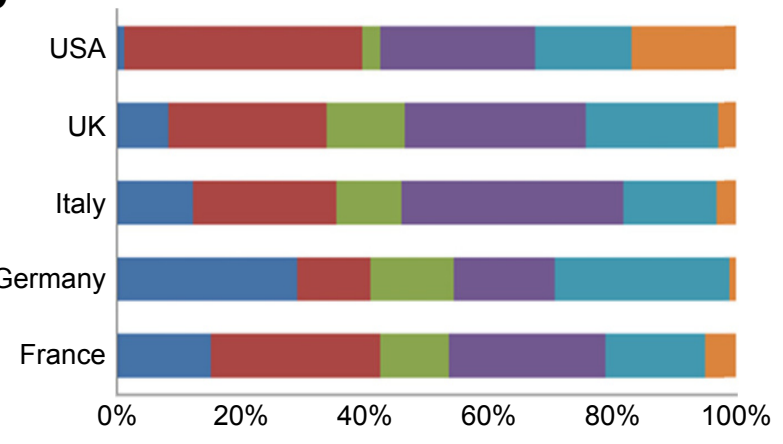

Platinum-based chemo $\square$ Non-platinum based chemo $\square$ Olaparib $\square$ Bevacizumab mono Bevacizumab + chemo $\square$ Other

Figure 2 Maintenance of BRCA-negative ovarian cancer patients.

Notes: Proportion of patients split by country and type of therapy: (A) first-line treatment, $(\mathbf{B})$ second-line treatment, $(\mathbf{C})$ third-line treatment, $(\mathbf{D})$ fourth-line treatment or more.

A

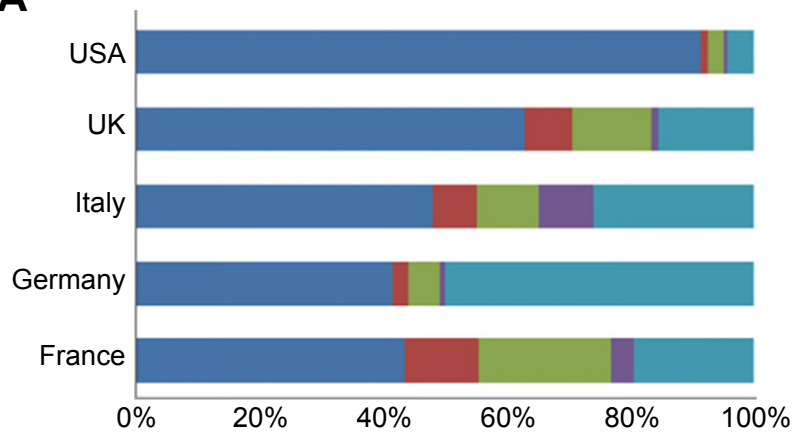

C

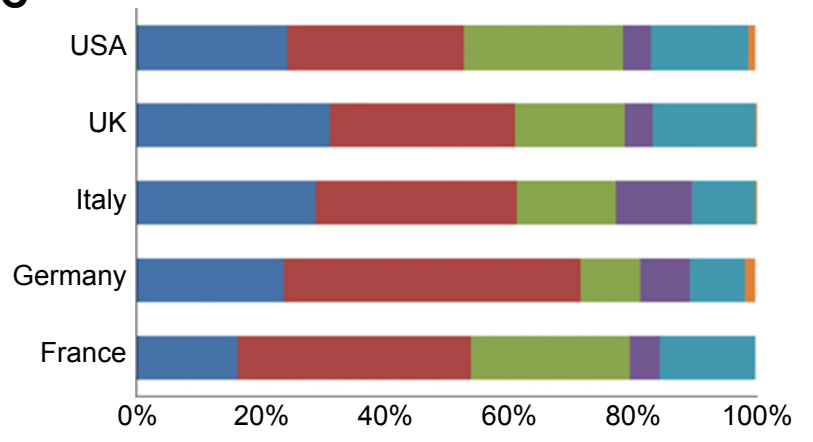

B

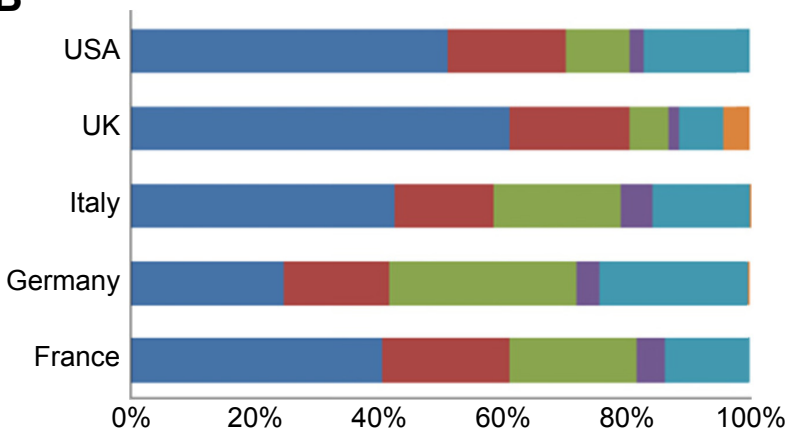

D

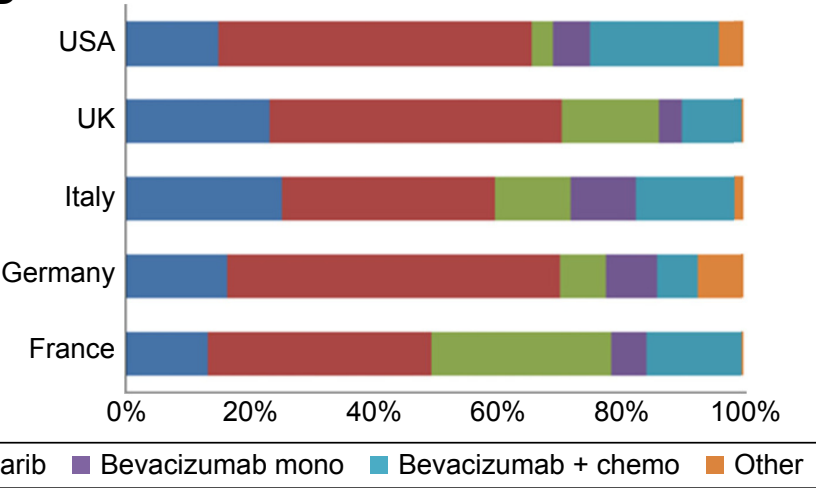

Figure 3 Treatment of BRCA-positive ovarian cancer patients.

Notes: Proportion of patients split by country and type of therapy: (A) first-line treatment, (B) second-line treatment, (C) third-line treatment, (D) fourth-line treatment or more. 

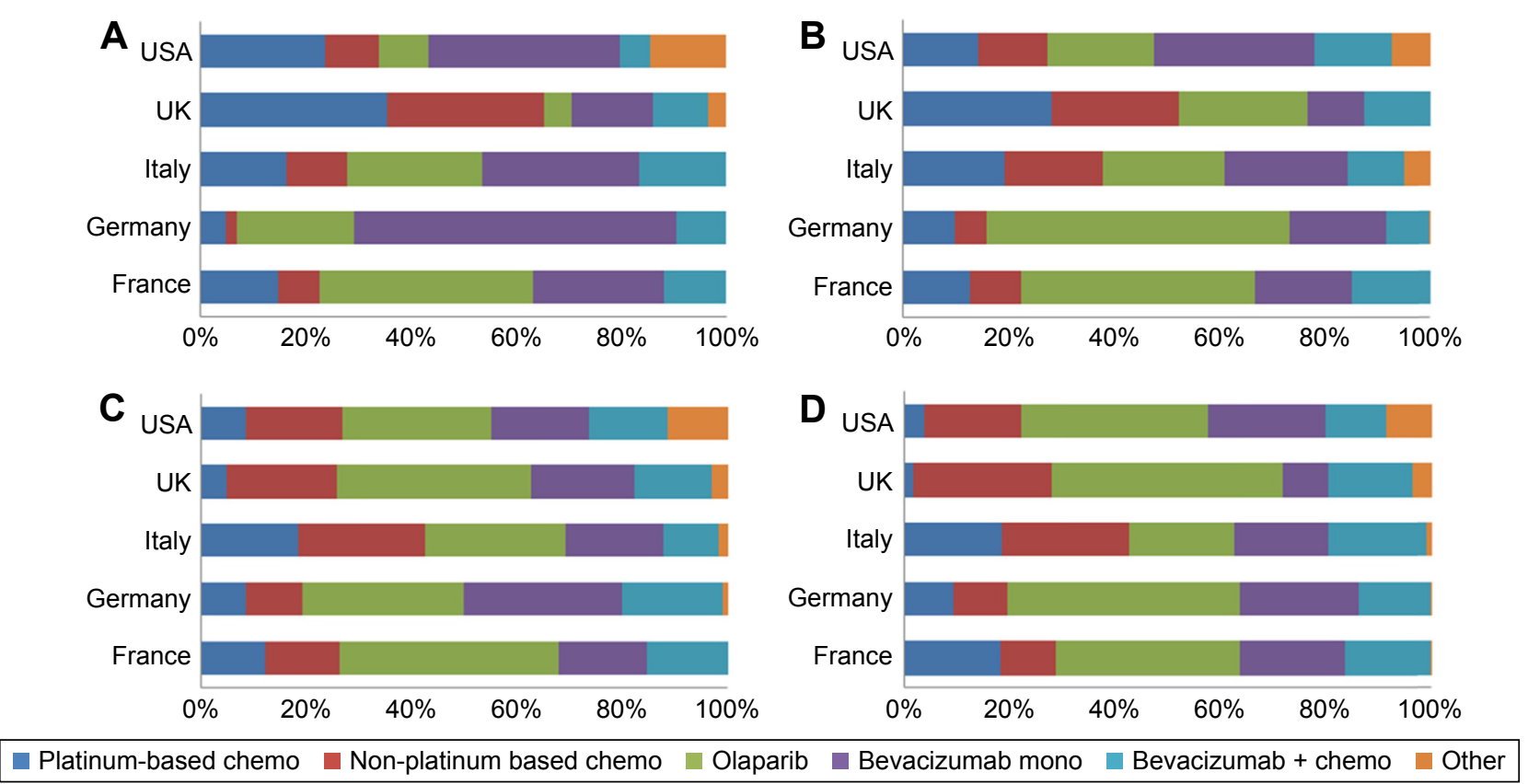

Figure 4 Maintenance of BRCA-positive ovarian cancer patients.

Notes: Proportion of patients split by country and type of therapy. (A) first-line treatment, (B) second-line treatment, (C) third-line treatment, (D) fourth-line treatment or more.

Our survey identified a consensus across countries regarding the proportion of advanced ovarian cancer patients receiving treatment versus those in maintenance therapy. More specifically, shares of patients in treatment, maintenance therapy, and remission were the same across lines of treatment. In all five surveyed countries, and for all lines of treatment, $60 \%$ of patients were in treatment, $20 \%$ in maintenance, and $20 \%$ in remission. Similarly, the emergence of platinum resistance was the same across countries.

We observed a number of country-specific differences for other aspects investigated in our survey. The proportion of $B R C A$-tested patients was highest in the USA at $73 \%$ and lowest in Italy and the UK at $45 \%$ and $50 \%$, respectively. Similarly, slight variations in the proportion of patients receiving neoadjuvant therapy were noted, with the highest proportion in France at 26\% and the lowest in Germany at $16 \%$. No specific reasons were identified for these regional differences.

Our survey highlights the magnitude of olaparib and bevacizumab prescription outside of their approved indication. Although olaparib was only indicated for $B R C A$-positive patients at the time of our survey, some prescribing to $B R C A$ negative patients in maintenance was reported, especially in Europe, and to a lesser extent in the treatment setting. The rationale for using olaparib in $B R C A$-negative patients was not investigated, and it is not known what proportion of patients had a positive outcome. Looking at $B R C A$-positive patients in the USA, where olaparib is indicated for the treatment of $B R C A$-positive patients who received three or more lines of treatment, our data identified some prescribing as early as first line, and in both the treatment and the maintenance setting. Similarly, in Europe, where olaparib was indicated in the maintenance setting only, some $B R C A$-positive patients received olaparib as treatment.

As for bevacizumab, its indication coverage was broad in Europe, where it can be used in front line, in the maintenance setting, and for both recurrent platinum-sensitive and platinum-resistant patients. As expected, we reported a broad range of bevacizumab prescribing, with roughly one-quarter of patients receiving it either in monotherapy or in combination with chemotherapy in the treatment setting, and one third in the maintenance setting. In the USA, where bevacizumab was indicated in the treatment of recurrent platinum-resistant patients in combination with chemotherapy at the time of our survey, our data confirmed that it was largely used as per its indication in the treatment setting, although we observed some off-label use in front line. However, bevacizumab was also largely used in monotherapy in the maintenance setting, which was outside of its indication in the USA.

We hypothesize that prescribing of bevacizumab and olaparib outside of their indications is driven by either offlabel use or inclusion in clinical trials. This is in line with the National Comprehensive Cancer Network guidelines that 
recommend enrolling advanced ovarian cancer patients who relapse on clinical trials as soon as possible. ${ }^{26}$

The poor prognosis in advanced ovarian cancer emphasizes the need for more options to be developed. The treatment algorithm for ovarian cancer is expected to continue to change with the approval of rucaparib by the FDA in December 2016 for the treatment of BRCA-positive ovarian cancer patients who have been treated with two or more chemotherapies, and the investigation of other targeted therapies such as niraparib. However, the true impact of these drugs on patient outcomes remains to be seen.

This survey has a number of limitations. Although this survey was distributed to a wide array of oncologists, this may not be a representative sample of ovarian cancer treating physicians as information about nonresponders was not collected. Therefore, caution should be used when generalizing results of this subset of oncologists to the entire advanced ovarian cancer treating physician population. As with any web-based survey, this study is susceptible to both selection bias and reporting bias. Importantly, all respondents answered the same survey questions. There were no differences in the questionnaires, outside of translation, regardless of whether respondents were recruited by the Deerfield Institute or the M3 panel.

\section{Conclusion}

Our survey illustrates the complexity of advanced ovarian cancer management and highlights country differences when it comes to the management of these patients. First, $B R C A$ testing was not performed systematically, which raises concerns regarding the access to the most appropriate treatment for the patients whose $B R C A$ status is unknown, especially in Italy and the UK. Second, although off-label prescription of targeted therapy was observed in all surveyed countries, there was no consensus regarding when these therapies could be used off label. Similarly, the preference for chemotherapeutic regimens or targeted therapy as per the disease stage varied in each country. These findings suggest the optimum usage of targeted therapies still needs to be determined.

\section{Acknowledgment}

Financial support for this research was provided by the Deerfield Institute, the internal research group at Deerfield Management Company, a health care investment firm dedicated to advancing health care through investment, information, and philanthropy.

\section{Disclosure}

The authors report no conflicts of interest in this work.

\section{References}

1. International Agency for Research on Cancer. GLOBOCAN 2012. Available from: http://gco.iarc.fr/today/online-analysis-multibars? mode $=$ cancer $\&$ mode_population $=$ continents $\&$ population $=$ $900 \&$ sex $=2 \&$ cancer $=29 \&$ type $=0 \&$ statistic $=0 \&$ prevalence $=0 \&$ color_palette=default. Accessed March 3, 2017.

2. Siegel RL, Miller KD, Jemal A. Cancer Statistics, 2017. CA Cancer J Clin. 2017;67(1):7-30.

3. Antoniou A, Pharoah PD, Narod S, et al. Average risks of breast and ovarian cancer associated with BRCA1 or BRCA2 mutations detected in case series unselected for family history: a combined analysis of 22 studies. Am J Hum Genet. 2003;72(5):1117-1130.

4. Chen S, Parmigiani G. Meta-analysis of BRCA1 and BRCA2 penetrance. J Clin Oncol. 2007;25(11):1329-1333.

5. Tsilidis KK, Allen NE, Key TJ, et al. Oral contraceptive use and reproductive factors and risk of ovarian cancer in the European Prospective Investigation into Cancer and Nutrition. Br J Cancer. 2011;105(9): 1436-1442.

6. Salani R, Backes FJ, Fung MF, et al. Post-treatment surveillance and diagnosis of recurrence in women with gynecologic malignancies: society of gynecologic oncologists recommendations. Am J Obstet Gynecol. 2011;204(6):466-478.

7. Wright AA, Bohlke K, Armstrong DK, et al. Neoadjuvant chemotherapy for newly diagnosed, advanced ovarian cancer: Society of Gynecologic Oncology and American Society of Clinical Oncology Clinical Practice Guideline. J Clin Oncol. 2016;34(28):3460-3473.

8. Jayson GC, Kohn EC, Kitchener HC, Ledermann JA. Ovarian cancer. Lancet. 2014;384(9951):1376-1388.

9. Kehoe S, Hook J, Nankivell M, et al. Primary chemotherapy versus primary surgery for newly diagnosed advanced ovarian cancer (CHORUS): an open-label, randomized, controlled, non-inferiority trial. Lancet. 2015;386(9990):249-257.

10. Trimbos JB, Parmar M, Vergote I, et al; European Organisation for Research and Treatment of Cancer Collaborators-Adjuvant ChemoTherapy un Ovarian Neoplasm. International collaborative ovarian neoplasm trial 1 and adjuvant chemotherapy in ovarian neoplasm trial: two parallel randomized phase III trials of adjuvant chemotherapy in patients with early-stage ovarian carcinoma. J Natl Cancer Inst. 2003; 95(2):105-112.

11. Trimbos JB, Vergote I, Bolis G, et al; EORTC-ACTION collaborators. European Organization for Research and Treatment of Cancer-Adjuvant ChemoTherapy in Ovarian Neoplasm. Impact of adjuvant chemotherapy and surgical staging in early-stage ovarian carcinoma: European organization for research and treatment of cancer-adjuvant chemotherapy in ovarian neoplasm trial. J Natl Cancer Inst. 2003;95(2):113-125.

12. Armstrong DK, Bundy B, Wenzel L, et al. Intraperitoneal cisplatin and paclitaxel in ovarian cancer. $N$ Engl J Med. 2006;354:34-43.

13. Katsumata N, Yasuda M, Isonishi S, et al; Japanese Gynecologic Oncology Group. Long-term results of dose-dense paclitaxel and carboplatin versus conventional paclitaxel and carboplatin for treatment of advanced epithelial ovarian, fallopian tube, or primary peritoneal cancer (JGOG 3016): a randomized, controlled, open-label trial. Lancet Oncol. 2013; 14(10):1020-1026.

14. Markman M, Liu PY, Moon J, et al. Impact on survival of 12 versus 3 monthly cycles of paclitaxel $(175 \mathrm{mg} / \mathrm{m} 2)$ administered to patients with advanced ovarian cancer who attained a complete response to primary platinum-paclitaxel: follow-up of a Southwest Oncology Group and Gynecologic Oncology Group phase 3 trial. Gynecol Oncol. 2009; 114(2):195-198.

15. FDA web site, Bevacizumab solution in combination with Paclitaxel. Available from: https://www.fda.gov/Drugs/InformationOnDrugs/ ApprovedDrugs/ucm423159.htm. Accessed March 17, 2017.

16. Kim G, Ison G, McKee AE, et al. FDA approval summary: olaparib monotherapy in patients with deleterious germline BRCA-mutated advanced ovarian cancer treated with three or more lines of chemotherapy. Clin Cancer Res. 2015;21(19):4257-4261. 
17. Lin YS, Nguyen C, Mendoza JL, et al. Preclinical pharmacokinetics, interspecies scaling, and tissue distribution of a humanized monoclonal antibody against vascular endothelial growth factor. J Pharmacol Exp Ther. 1999;288(1):371-378.

18. Aghajanian C, Goff B, Nycum LR, Wang YV, Husain A, Blank SV. Final overall survival and safety analysis of OCEANS, a phase III trial of chemotherapy with or without bevacizumab in patients with platinum-sensitive recurrent ovarian cancer. Gynecol Oncol. 2015; 139(1):10-16.

19. Pujade-Lauraine E, Hilpert F, Weber B, et al. Bevacizumab combined with chemotherapy for platinum-resistant recurrent ovarian cancer: The AURELIA open-label randomized phase III trial. J Clin Oncol. 2014;32(13):1302-1308.

20. Burger RA, Brady MF, Bookman MA, et al; Gynecologic Oncology Group. Incorporation of bevacizumab in the primary treatment of ovarian cancer. $N$ Engl J Med. 2011;365(26):2473-2483.

21. Oza AM, Cook AD, Pfisterer J, et al. Standard chemotherapy with or without bevacizumab for women with newly diagnosed ovarian cancer (ICON7): overall survival results of a phase III randomized trial. Lancet Oncol. 2015;16(8):928-936.
22. Emile G, Chauenet L, Tigaud JM, et al. A clinical experience of single agent bevacizunab in relapsing ovarian cancer. Gynecol Oncol. 2013; 129(3):459-462.

23. Fong PC, Boss DS, Yap TA, et al. Inhibition of poly (ADP-ribose) polymerase in tumors from BRCA mutation carriers. $N$ Engl J Med. 2009; 361(2):123-134.

24. OlaparibEPAR onEMA web site. Available from: http://www.ema.europa. eu/ema/index.jsp?curl=pages/medicines/human/medicines/003726/ human_med_001831.jsp\&mid=WC0b01ac058001d124. Accessed March 17, 2017.

25. US Department of Health and Human Services. Protection of human subjects 45 CFR $\$ 46.101$, paragraph (b) (4) General requirements for informed consent. Available from: https:/www.hhs.gov/ohrp/ regulations-and-policy/regulations/45-cfr-46/index.html\#46.116. Accessed March 17, 2017.

26. National Comprehensive Cancer Network web site, Ovarian cancer guidelines. Available from: https://www.nccn.org/professionals/ physician_gls/pdf/ovarian.pdf. Accessed April 7, 2017. 


\section{Supplementary materials}

A

\begin{tabular}{|c|c|c|c|}
\hline USA & $62 \%$ & $15 \%$ & $23 \%$ \\
\hline UK & $50 \%$ & $21 \%$ & $29 \%$ \\
\hline Italy & $57 \%$ & $25 \%$ & $18 \%$ \\
\hline Germany & $61 \%$ & $21 \%$ & $18 \%$ \\
\hline France & $59 \%$ & $24 \%$ & $17 \%$ \\
\hline
\end{tabular}

C

\begin{tabular}{|c|c|c|c|}
\hline USA & $62 \%$ & $16 \%$ & $21 \%$ \\
\hline UK & $59 \%$ & $16 \%$ & $25 \%$ \\
\hline Italy & $53 \%$ & $24 \%$ & $22 \%$ \\
\hline Germany & $64 \%$ & $20 \%$ & $16 \%$ \\
\hline France & $63 \%$ & $21 \%$ & $16 \%$ \\
\hline
\end{tabular}

B

\begin{tabular}{|c|c|c|c|}
\hline USA & $61 \%$ & $19 \%$ & $20 \%$ \\
\hline UK & $55 \%$ & $18 \%$ & $27 \%$ \\
\hline Italy & $57 \%$ & $23 \%$ & $20 \%$ \\
\hline Germany & $65 \%$ & $21 \%$ & $14 \%$ \\
\hline France & $65 \%$ & $21 \%$ & $14 \%$ \\
\hline
\end{tabular}

D

\begin{tabular}{|c|c|c|c|c|}
\hline USA & $59 \%$ & & $19 \%$ & $22 \%$ \\
\hline UK & $62 \%$ & & $15 \%$ & $23 \%$ \\
\hline Italy & $44 \%$ & $25 \%$ & & $31 \%$ \\
\hline Germany & $71 \%$ & & & $7 \% \quad 13 \%$ \\
\hline France & $58 \%$ & & $25 \%$ & $17 \%$ \\
\hline
\end{tabular}

- Treatment $\quad$ Maintenance $\quad$ Patient in remission

Figure SI Proportion of patients as per the type of management in each country.

Notes: (A) First-line treatment, (B) second-line treatment, (C) third-line treatment, (D) fourth line or above. Based on 37 oncologists in France, 37 in Germany, 3 I in Italy, 33 in the UK, and 132 in the USA.

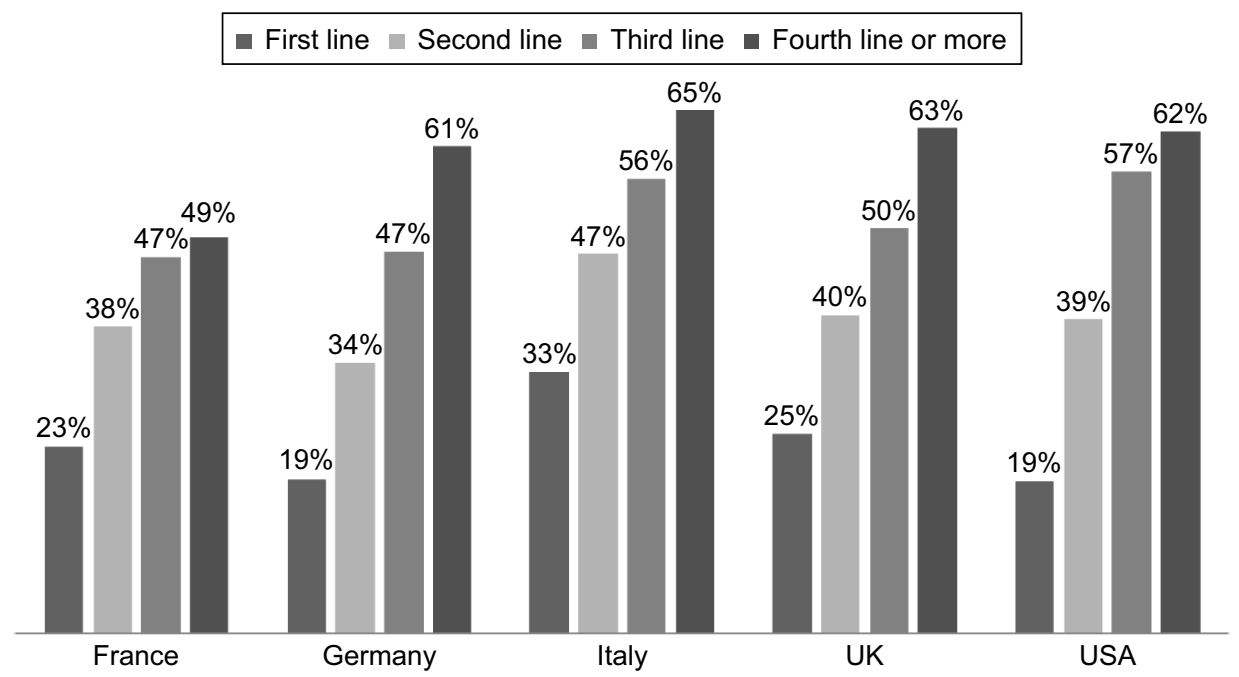

Figure S2 Percentage of patients who are refractory or resistant to platinum-based chemotherapy, as per the country and the line of therapy. Notes: Based on 37 oncologists in France, 37 in Germany, 31 in Italy, 33 in the UK, and I 32 in the USA.

\section{Publish your work in this journal}

Drug Design, Development and Therapy is an international, peerreviewed open-access journal that spans the spectrum of drug design and development through to clinical applications. Clinical outcomes, patient safety, and programs for the development and effective, safe, and sustained use of medicines are the features of the journal, which has also been accepted for indexing on PubMed Central. The manuscript management system is completely online and includes a very quick and fair peer-review system, which is all easy to use. Visit http://www.dovepress.com/testimonials.php to read real quotes from published authors.

Submit your manuscript here: http://www.dovepress.com/drug-design-development-and-therapy-journal 